\title{
ON ESSENTIALLY ABSOLUTELY CONTINUOUS PLANE TRANSFORMATIONS
}

\author{
TIBOR RADÓ
}

1. Introduction. Let $Q$ be the unit square $0 \leqq u \leqq 1,0 \leqq v \leqq 1$, in the $u v$-plane $(u, v$ are Cartesian coordinates), and let $x(u, v), y(u, v)$ be real-valued continuous functions in $Q$. These functions determine a continuous mapping $T: x=x(u, v), y=y(u, v),(u, v) \in Q$, from $Q$ into the $x y$-plane, where $x, y$ are again Cartesian coordinates. If we introduce, for convenience, the complex variables $w=u+i v, z=x+i y$, then $T$ appears in the form

$$
T: \quad z=f(w), \quad w \in Q,
$$

where $f(w)=x(u, v)+i y(u, v)$. In connection with various problems in geometry and in analysis, there arises the problem of defining the concepts of bounded variation and of absolute continuity for continuous mappings $T$ given as in (1). A detailed review of the extensive relevant literature may be found in Chapter IV.5 of the writer's book Length and area. This book will be referred to as LA, and will be used as general reference both for technical details and for literature. The present note is concerned with a line of thought that led to the concepts $\mathrm{eBV}$ (essential bounded variation) and $\mathrm{eAC}$ (essential absolute continuity). The theory of these concepts, as presented in LA, is based on the work of the writer and P. V. Reichelderfer. During the war years, Cesari in Italy developed an analogous theory based on apparently different basic concepts. However, as the writer has shown (see Bibliography [2]), the basic concepts used by Cesari are equivalent to those used in LA (see Bibliography). Thus the theories developed in LA and in the work of Cesari respectively can be combined into a single theory whose aim it is to provide two-dimensional concepts of bounded variation and absolute continuity comparable in scope and in utility to the corresponding classical one-dimensional concepts for functions of a single real variable. The present status of the theory seems to justify the assumption that the two-dimensional concepts $\mathrm{eBV}$ and $\mathrm{eAC}$ (see above) represent adequate generalizations of the corresponding one-dimensional concepts. However, it seems desirable to put the definitions of the two-dimensional concepts into a form which reveals the fundamental analogy with the one1948.

Presented to the Society, December 31, 1947; received by the editors April 26, 
dimensional concepts as clearly as possible. The purpose of the present note is to make a contribution in this direction for the concept eAC.

2. The one-dimensional situation. Let $I$ denote the unit interval $0 \leqq u \leqq 1$ on a $u$-axis, and let

$$
T: \quad x=f(u), \quad u \in I,
$$

be a continuous mapping from $I$ into a (real) $x$-axis. Naturally, $T$ is then termed BV (of bounded variation) or AC (absolutely continuous) if and only if the function $f(u)$ is BV or AC respectively. However, the geometrical picture yields an important re-wording as follows. Let us define a multiplicity function $N(x)$ by the agreement: for $-\infty<x_{0}<+\infty, N\left(x_{0}\right)$ is equal to the number (possibly infinite) of those points $u \in I$ that are mapped by $T$ into $x_{0}$. The following facts are well known.

(a) $T$ is $\mathrm{BV}$ if and only if $N(x)$ is summable.

(b) If $T$ is $\mathrm{BV}$, then it is $\mathrm{AC}$ if and only if for every set $E$ of measure zero in $I$ the image set $T(E)$ is also of measure zero.

3. The two-dimensional situation. In dealing with the two-dimensional situation, we shall use LA as a reference. However, in LA a more general situation is considered, namely the case where the mapping $T$ operates from a general bounded domain. In the special case considered in this note, the domain is taken as the interior of the unit square $Q$, and $T$ is assumed to be defined and continuous on the perimeter also. These restrictions are made only to simplify the presentation, and our main result is readily seen to remain valid in the general case considered in LA.

Given $T$ as in (1), there is associated with $T$ an essential multiplicity function $\kappa(z, T, Q)$ (see LA, IV.1.4). Furthermore, with each point $z$ there is associated a class (possibly empty or infinite) of continua in the interior $Q^{0}$ of $Q$, namely the class of the essential maximal model continua (abbreviated to e.m.m.c.) of $z$ under $T$ in $Q$ (see LA IV.1.27). As a matter of fact, $\kappa(z, T, Q)$ is equal to the number of the e.m.m.c.'s of $z$ (see LA IV.1.39). The sum of all the e.m.m.c.'s, corresponding to all the points $z$, is the complete essential set $\mathcal{E} *$, while the restricted essential set $\varepsilon$ is the sum of all those e.m.m.c.'s that reduce to single points (see LA IV.1.56). Both $\varepsilon^{*}$ and $\varepsilon$ are Borel sets (see LA IV.1.58, IV.1.59). In analogy with the statements (a), (b) in $\S 2$ above, we have the following statements in the two-dimensional case.

(a) The mapping $T$, given as in (1), is $e B V$ if and only if the essen- 
tial multiplicity function $\kappa(z, T, D)$ is summable.

(b) If $T$ is $e B V$, then it is $e A C$ if and only if for every set $E$ of measure zero in the complete essential set $\mathcal{E}^{*}$ the image set $T(E)$ is also of measure zero.

The statement (a) is in fact the definition of the concept eBV (see LA, IV.4.1). The statement (b) is the new result that we propose to prove in the present note.

4. Background. In an interesting paper on absolutely continuous mappings, L. Giuliano (see Bibliography [1]) established the following facts: If the mapping $T$, given as in (1), is eBV and maps subsets of measure zero of $Q^{0}$ into sets of measure zero, then $T$ is eAC. However, examples show that this condition is not necessary for $T$ to be eAC.

Thus it would seem that the characterization (b) of AC mappings in the one-dimensional case (see \$2) admits of no analogue for the two-dimensional eAC concept. And yet, our theorem (b) in $\$ 3$ reveals a fundamental analogy. This theorem also reveals the importance of the complete essential set $\varepsilon^{*}$. The basic importance of the complete essential set $\mathcal{E}^{*}$, as against the restricted essential set $\mathcal{E}$, was first recognized by P. V. Reichelderfer (see Bibliography [3]).

5. Proof of the theorem. Necessity. Suppose that $T$, given as in (1), is eAC. By definition (see LA IV.4.1) $T$ is then AC $\mathcal{E}^{*}$ (see LA IV.2.39). Hence, by LA IV.2.42, for every set $E$ of measure zero in $\mathcal{E}^{*}$ the image set $T(E)$ is of measure zero.

Sufficiency. Suppose that $T$, given as in (1), satisfies the following conditions.

(i) $T$ is eBV (see LA IV.4.1).

(ii) For every set $E$ of measure zero in $\mathcal{E}^{*}$, the image set $T(E)$ is of measure zero.

We have to show that $T$ is then eAC. To this end, we verify the following fact.

(iii) $T$ is BVE* (see LA IV.2.11).

Indeed, by LA IV.4.1, IV.2.42, the statements (ii) and (iii) imply that $T$ is eAC, and hence it is sufficient to verify (iii). Now, by LA IV.2.13, the statement (iii) is equivalent to the following fact.

(iv) the function $N\left(z, \mathcal{E}^{*}\right)$ is summable (where $N\left(z, \mathcal{E}^{*}\right)$ is the number, possibly infinite, of those points $w \in \mathcal{E}^{*}$ whose image under $T$ is $z)$.

To establish (iv), we note that by assumption (i) the essential multiplicity function $\kappa(z, T, Q)$ is summable (see LA IV.4.1). Hence (iv) will be proved if we can prove the following fact. 
(v) $N\left(z, \mathcal{E}^{*}\right)=\kappa(z, T, Q)$ a.e. (almost everywhere) in the $z$-plane.

To establish (v), let us denote for $0 \leqq r \leqq 1, r$ rational, by $E_{r}^{\prime}$ the intersection of $\varepsilon^{*}$ with the line $u=r$, and by $E_{r}^{\prime \prime \prime}$ the intersection of $\varepsilon^{*}$ with the line $v=r$. Then $E_{r}^{\prime}$, for fixed $r$, is a set of measure zero in $\mathcal{E}^{*}$, and hence by (ii) the image set $T\left(E_{r}^{\prime}\right)$ is of measure zero. Similarly the image set $T\left(E_{r}^{\prime \prime}\right)$ is of measure zero. Let us denote by $\bar{e}$ the set of those points $z$ where $\kappa(z, T, Q)=\infty$. Since $\kappa(z, T, Q)$ is summable, $\bar{e}$ is of measure zero. Let us put

$$
\bar{E}=\bar{e}+\sum_{r} T\left(E_{r}^{\prime}\right)+\sum_{r} T\left(E_{r}^{\prime \prime}\right)
$$

where the summations are extended over all rational numbers $r$ such that $0<r<1$. Obviously

$$
|\bar{E}|=0 \text {, }
$$

where $|\bar{E}|$ denotes the measure of $\bar{E}$. Now take any point $z_{0} \notin \bar{E}$. Then $z_{0} \notin \bar{e}$, and hence $\kappa\left(z_{0}, T, Q\right)=k_{0}$ is finite. Furthermore, if $c_{0}$ is any e.m.m.c. of $z_{0}$, then by the definition of $\bar{E}$ it follows that $c_{0}$ does not intersect any one of the lines $u=r$ or $v=r$, where $r$ is any rational number such that $0<r<1$. Hence $c_{0}$ must reduce to a single point. It follows that $N\left(z_{0}, \mathcal{E}^{*}\right)=k_{0}=\kappa\left(z_{0}, T, Q\right)$ (cf. LA IV.1.39). Hence

$$
N\left(z, \varepsilon^{*}\right)=\kappa(z, T, Q) \quad \text { for } z \notin \bar{E} \text {. }
$$

Clearly (4) and (5) imply (v), and thus the proof of our theorem (b) in $\$ 3$ is complete.

\section{BiBLIOGRAPHY}

LA. T. Radó, Length and area, Amer. Math. Soc. Colloquium Publications, vol. 30, 1948.

1. L. Giuliano, Sulle trasformazioni assolutamente continue, Annali Scuola Normale Superiore Pisa (2) vol. 12 (1943) pp. 161-172 (1947).

2. T. Radó, Two-dimensional concepts of bounded variation and absolute continuity, Duke Math. J. vol. 14 (1947) pp. 587-608.

3. P. V. Reichelderfer, On bounded variation and absolute continuity for parametric representations of continuous surfaces, Trans. Amer. Math. Soc. vol. 53 (1943) pp. 251291.

The OHio State University 